\title{
A Symmetry Connecting the Shell, Collective and Cluster Models
}

\author{
J. Cseh \\ Institute for Nuclear Research, Debrecen, Bem ter 18/C, Hungary-4026 \\ Received 6 October 2021 \\ doi: https://doi.org/10.55318/bgjp.2021.48.5-6.457
}

\begin{abstract}
The relation of the fundamental structure models is discussed. Their common intersection is provided by the U(3) dynamical symmetry for the single shell problem, and by the multiconfigurational dynamical symmetry (MUSY) of group structure $\mathrm{U}_{s}(3) \otimes \mathrm{U}_{e}(3) \supset \mathrm{U}(3)$ $\supset \mathrm{SU}(3) \supset \mathrm{SO}(3)$ for the major-shell-excitation. Both of them show dual breaking: U(3) and SU(3) of the total Hamiltonian are dynamically broken, while $\mathrm{SO}(3)$ of the intrinsic Hamiltonian is spontaneously broken, resulting in deformed shape. MUSY has a composite nature: a simple dynamical symmetry is present in each configuration, and a further symmetry transforms them into each other. Together with the antisymmetrization it can completely wash out the difference between the model pictures, thus occasionally cluster and shell wavefunctions can be identical.
\end{abstract}

KEY WORDS: Multiconfigurational dynamical symmetry, structure models.

\section{Introduction}

The fundamental models of nuclear structure are based on different physical pictures. In the shell model we think the nucleus like a small atom, and the relevant degrees of freedom are those of the nucleons. The collective model treats it as a microscopic liquid drop, and applies shape parameters. The cluster model says it is similar to a molecule, and the important dynamics is the relative motion of the clusters. Due to this big variety, it is an interesting and important question, how these models are related to each other, and what is their common intersection, if there is any.

The basic answer was obtained in 1958 [1-3]. In the present day language we can say that the $\mathrm{U}(3) \supset \mathrm{SU}(3) \supset \mathrm{SO}(3)$ dynamical symmetry was found to be the common intersection of the three models for a single-shell problem. 
As for the multi-shell problem, no concise answer was available for a long time, in spite of the much valuable contribution from various approaches.

Here we present a symmetry-based connection among these fundamental structure models for the multi-major-shell case, which is an extension of the $\mathrm{U}(3)$ relation from 1958. It is called multiconfigurational dynamical symmetry, and it is based on the algebraic structure of the chain $\mathrm{U}_{s}(3) \otimes \mathrm{U}_{e}(3) \supset \mathrm{U}(3) \supset \mathrm{SU}(3)$ $\supset \mathrm{SO}(3)$.

In what follows, first (in Section 2) we recall some basic features of the development in 1958, and pay special attention to the relation of the shapes and symmetries (even if some aspects were revealed by later studies). Section 3 introduces MUSY, and presents its basic characteristics. Finally (in Section 4) a brief summary is given, and some conclusions are drawn.

\section{The 1958 Connection}

\subsection{Basic relations}

Elliott invented a spherical shell model [1] with harmonic oscillator potential and quadrupole residual interaction of the nucleons:

$$
H=H_{H O}+\chi Q Q
$$

where $Q$ is the sum of the nucleon quadrupole moments. This equation can be rewritten in an algebraic form:

$$
H=C_{U 3}^{(1)}+\alpha C_{S U 3}^{(2)}+\beta C_{S O 3}^{(2)},
$$

where $C^{(i)}$ is the invariant operator of degree $i$ of the algebra indicated in the subscript. Since it is expressed in terms of Casimir operators of a single algebrachain

$$
\begin{array}{cl}
\mathrm{U}(3) \quad \supset \mathrm{SU}(3) \supset \mathrm{SO}(3) \supset & \mathrm{SO}(2) \\
{\left[n_{1}, n_{2}, n_{3}\right],(\lambda, \mu), K, \quad L, \quad} & M .
\end{array}
$$

its eigenvalue-problem has an analytical solution. This situation is called (broken) dynamical symmetry, and it plays important role in many algebraic models. (Here we have indicated also the representation labels.) The spin-isospin sector of the model space can be described by Wigner's supermultiplet theory [4] of $\mathrm{U}^{\mathrm{ST}}(3) \supset \mathrm{U}^{\mathrm{S}}(2) \otimes \mathrm{U}^{\mathrm{T}}(2)$, group structure.

Elliott showed that the quadrupole shape of the nucleus is determined by the $(\lambda, \mu) \mathrm{SU}(3)$ quantum numbers. E.g. $(0,0)$ is spherical $(\lambda, 0)$ is prolate $(0, \mu)$ is oblate, and in general $(\lambda, \mu)$ has a triaxial shape. He also revealed that a rotational band is characterised by a well-defined $\mathrm{SU}(3)$ symmetry. 
In the same year Wildermuth and Kanellopoulos showed [2] that the Hamiltonian of the shell model can be rewritten exactly into that of the cluster model in the harmonic oscillator approximation

$$
H_{S M}=H_{C M}
$$

This relation builds up, of course, a very close connection between the wavefunctions of the two models, too. In particular, a cluster model state is obtained as a linear combination of those shell model basis states that belong to the same energy.

Bayman and Bohr succeeded to reinterpret the cluster-shell [3] connection in terms of the U(3) symmetry. Therefore, in 1958 specific collective and cluster bands could be picked up from the see of the shell model states by their welldefined SU(3) symmetries.

This beautiful connection of the fundamental structure models was found for a single major-shell problem.

\subsection{Shape and symmetry}

The quantitative relation by which the $S U(3)$ symmetry determines the quadrupole deformation is $[5,6]$ :

$$
\beta^{2}=\frac{16 \pi}{5 N_{0}^{2}}\left(\lambda^{2}+\mu^{2}+\lambda \mu\right), \gamma=\arctan \left(\frac{\sqrt{3} \mu}{2 \lambda+\mu}\right) .
$$

Here $N_{0}$ is the number of oscillator quanta, including the zero point contribution: $N_{0}=n+(A-1) \frac{3}{2}, n$ is the sum of the $\mathrm{U}(3)$ quantum numbers: $n=n_{1}+n_{2}+$ $n_{3}$, and $A$ is the mass number of the nucleus.

The deformation of the nuclear ground state is an example of the spontaneous symmetry breaking. Simply speaking a symmetry is spontaneously broken, if the Hamiltonian shows the symmetry, but the ground state of the system does not. This is a typical case with the rotational symmetry in nuclei. The phenomenon has been discussed both in the mean-field approach [7,8], and in the interacting boson model $[9,10]$. Interestingly enough, however, it was not addressed within the framework of the Elliott model until recently. This is somewhat surprising, considering the fact that it was the first model to connect the quadrupole deformation (and the collective rotation) to the spherical shell model, and it is also the prototype of the algebraic structure models. In fact it offers a very simple and transparent way [11] for the illustration of the spontaneous breaking.

The model Hamiltonian of Eq. (2) can be split up exactly into an intrinsic (i.e. fast) and collective (i.e. slow) parts:

$$
H=H_{\mathrm{intr}}+H_{\mathrm{coll}}, \quad H_{\mathrm{intr}}=C_{U 3}^{(1)}+\alpha C_{S U 3}^{(2)}, \quad H_{\mathrm{coll}}=\beta C_{S O 3}^{(2)} .
$$


$H_{\text {intr }}$ determines the energy of the band-heads, and $H_{\text {coll }}=\beta L L$ splits up the bands. Not only the total $H$, but also $H_{\text {intr }}$ is rotationally invariant, yet the intrinsic shape of the nucleus may be deformed, as a result of the spontaneous breaking in the eigenvalue-problem of $H_{\text {intr }}$. It happens when the ground state of $H_{\text {intr }}$ is degenerate, i.e. more than one state has the same energy. The spurious excitation between these degenerate states correspond to the Goldstone bosons [11], which appear, when a continuous global symmetry is spontaneously broken [12].

The same kind of spontaneous breaking takes place in many other algebraic structure models; some of them are direct extensions of the Elliott model [11].

The scenario we have observed here is a typical one for the spontaneous symmetry breaking, not only in nuclear physics [13]. In particular:

1. The Hamiltonian is separated into a fast and slow parts.

2. The symmetry of the fast part breaks spontaneously, when there is a degenerecy.

3. The symmetry recovers when all the degrees of freedom are taken into account, i.e. the complete Hamiltonian is considered.

In light of the previous paragraphs we can say that in the Elliott model a dual symmetry breaking shows up. The U(3) and SU(3) symmetries are dynamically broken by the interactions expressed in terms of the invariants of their subalgebras (i.e. $\mathrm{SU}(3)$ and $\mathrm{SO}(3)$ ), while the rotational symmetry is spontaneously broken in the eigenvalue problem of the intrinsic part of the Hamiltonian. This kind of dual symmetry breaking is also typical in algebraic models of nuclear structure [14].

\section{Multi-Shell Connection: MUSY}

For the multi-shell problem the connecting symmetry is characterized by the group-chain:

$$
\begin{aligned}
& \mathrm{U}_{\mathrm{s}}(3) \otimes \quad \mathrm{U}_{\mathrm{e}}(3) \quad \supset \mathrm{U}(3) \supset \mathrm{SU}(3) \supset \mathrm{SO}(3) \\
& \left|\left[n_{1}^{s}, n_{2}^{s}, n_{3}^{s}\right],\left[n_{1}^{e}, n_{2}^{e}, n_{3}^{e}\right], \rho,\left[n_{1}, n_{2}, n_{3}\right],(\lambda, \mu), K, L\right\rangle \text {. }
\end{aligned}
$$

One arrives at this structure by investigating the algebraic models of the multishell problem from the shell, collective and cluster sides, as shown below.

This symmetry is a composite symmetry in the sense that each configuration has a simple $\mathrm{U}(3)$ dynamical symmetry, and there is a further symmetry connecting the configurations to each other. The logical structure is similar to that of the dynamical supersymmetry (SUSY) of nuclear physics. In the SUSY case a system is considered, which has a bosonic and a fermionic sector, each of them equipped with a dynamical symmetry, and the supertransformations connect the two sectors. 
A Symmetry Connecting the Shell, Collective and Cluster Models

\subsection{Shell model}

From the shell model perspective a successful extension of the Elliott model is the symplectic model $[15,16]$. It includes major shell excitations of 2 oscillator quanta, connecting shells of the same parity. Many physical phenomena requires shell excitation, e.g. the calculation of the E2 transitions. In the Elliott model it is described by applying an effective charge, i.e. a phenomenological parameter. The description with real electric charges requires shell excitations. This model has a symplectic group-structure, together with its subgroups:

$$
\begin{gathered}
\mathrm{Sp}(6, \mathrm{R}) \longrightarrow \mathrm{U}(3) \supset \mathrm{SU}(3) \supset \mathrm{SO}(3) \\
\left|\left[n_{1}^{s}, n_{2}^{s}, n_{3}^{s}\right],\left[n_{1}^{e}, n_{2}^{e}, n_{3}^{e}\right], \rho,\left[n_{1}, n_{2}, n_{3}\right],(\lambda, \mu), K, L\right\rangle .
\end{gathered}
$$

Here $\left[n_{1}^{s}, n_{2}^{s}, n_{3}^{s}\right]$ denotes the symplectic bandhead, which is a U(3) irrep, being a lowest-weight $\operatorname{Sp}(6, \mathrm{R})$ state. $\left[n_{1}^{e}, n_{2}^{e}, n_{3}^{e}\right]$ corresponds to the major-shell excitations. Please, note that the basis states are characterized by the irreps of group-chain (7). The symplectic model is a multi-major-shell extension of the Elliott model on one hand, and it is a microscopic collective model on the other hand, including collective rotation and vibration.

\subsection{Collective model}

The symplectic model has a simplified version, called contracted symplectic model $[17,18]$. From the mathematical viewpoint it is simpler, because it has a compact algebraic structure of $\mathrm{U}_{s}(3) \otimes \mathrm{U}_{b}(6)$ (as opposed to the noncompact $\mathrm{Sp}(6, \mathrm{R}))$, and an orthonormal set of basis:

$$
\mathrm{U}_{\mathrm{s}}(3) \otimes \mathrm{U}_{\mathrm{e}}(6) \supset \mathrm{U}_{\mathrm{s}}(3) \otimes \mathrm{U}_{\mathrm{e}}(3) \supset \mathrm{U}(3) \supset \mathrm{SU}(3) \supset \mathrm{SO}(3),
$$

including the $\mathrm{U}_{\mathrm{s}}(3) \otimes \mathrm{U}_{\mathrm{e}}(3) \supset \mathrm{U}(3) \supset \mathrm{SU}(3) \supset \mathrm{SO}(3)$ part again. Physically the simplification is due to the bosonization, not fully including the Pauli principle. This model is an algebraic model of collective motion for a multimajor-shell problem.

\subsection{Cluster model}

As for the clusterization is concerned, the relevant approach for revealing the connection to other structure models needs to be algebraic and (semi)microscopic. The algebraic nature is obvious from the $\mathrm{U}(3)$-connection of the single-shell problem. The U(3) symmetry has been around in cluster studies from 1958, but only for the basis states. The fully algebraic description started in 1981, when Iachello invented the vibron model of U(4) group structure for dipole degrees of freedom [19]. This is what we need for the treatment of the relative motion of the clusters. Fully algebraic model means that not only the 
basis states, but also the physical operators carry group symmetries, like in the Elliott model e.g.

The microscopic nature is required for the model space, i.e. it has to appreciate the Pauli-principle. From the phenomenological viewpoint the shell and cluster configurations are orthogonal to each other, therefore, this approach is not suitable for the study of their relation.

The semimicroscopic algebraic cluster model (SACM) [20,21] combines a fully microscopic model space with a fully algebraic description. It describes the internal structure of the clusters by the Elliott model, and their relative motion by the vibron model, which is modified in order to incorporate the Pauli principle. A binary cluster configuration has a group structure of

$$
\begin{array}{r}
\mathrm{U}_{\mathrm{C}_{1}}(3) \otimes \mathrm{U}_{\mathrm{C}_{2}}(3) \otimes \mathrm{U}_{\mathrm{R}}(4) \supset \mathrm{U}_{\mathrm{C}}(3) \otimes \mathrm{U}_{\mathrm{R}}(3) \supset \\
\mathrm{U}(3) \supset \mathrm{SU}(3) \supset \mathrm{SO}(3),
\end{array}
$$

where $\mathrm{C}_{1}$, and $\mathrm{C}_{2}$ stand for individual clusters, $\mathrm{C}$ is for their coupled structure, and $\mathrm{R}$ indicates relative motion. Please, note again the presence of the groupchain (7).

In the models of the last three subsections we were dealing only with the space degrees of freedom, and did not discuss the spin-isospin sector. It can be described in each case by Wigner's supermultiplet theory of $\mathrm{U}^{\mathrm{ST}}(4) \supset$ $\mathrm{U}^{\mathrm{S}}(2) \otimes \mathrm{U}^{\mathrm{T}}(2)$, just like in the Elliott model. The antisymmetric requirement of the total wavefunction puts a constraint on the relation between the irreducible representation of $\mathrm{U}^{\mathrm{ST}}(4)$ and $\mathrm{U}(3)$, which is taken into account in each major shell separately, and then they are combined [22].

\subsection{Connecting symmetry: MUSY}

As the preceding subsections have shown it, the group-chain (7) provides us with a common classification scheme for the shell, collective and cluster models. The states are specified by the labels of irreducible representations of the same groups, the difference between them is the set of specific quantum numbers. This unified multiplet structure of the three models is the first pillar of MUSY [22].

It also needs a second one, i.e. transformations which take the different configurations into each other. In order to find them we need to consider another classification scheme. The one which has lead to chain (7) is based on the shell scheme in the sense that the model space is constructed in each major shell separately, and then they are combined. On the other hand the transformation from e.g. a shell configuration to a cluster one or vice versa, or between different clusterizations, requires the redistribution of the nucleons in the pseudo space of the particle indices. Such a scheme, that deals with the particles democratically, independent of their shell-association, have been worked out by Kramer and Moshinsky [23]. 


\section{A Symmetry Connecting the Shell, Collective and Cluster Models}

They have considered the problem of $\mathrm{n}$ nucleons in the 3 dimensional harmonic oscillator potential. This system has an $\mathrm{U}(3 \mathrm{n})$ symmetry group, and its subgroups: $\mathrm{U}(3)$ in the real space and $\mathrm{U}(\mathrm{n})$ in the particle index space $\mathrm{U}(3 \mathrm{n}) \supset$ $\mathrm{U}(3) \otimes U(n)$. The generators of these groups are the number conserving bilinear products of the oscillator quantum creation and annihilation operators. In particular, there are $3 n \times 3 n$ of them for $U(3 n), 3 \times 3$ for $U(3)$ and $n x n$ for $U(n)$. The generators of the subgroups are obtained from those of $\mathrm{U}(3 n)$ by contraction, i.e. summing up according to the particle indices for $\mathrm{U}(3)$, and according to the space indices for $\mathrm{U}(\mathrm{n})[22,23]$.

This classification scheme guides us in building up our formalism is such a way that it can be invariant with respect to the transformations in the pseudo space of particle indices. In particular, if we apply physical operators, which are expressed in terms of the generators of $\mathrm{U}(3)$ and its subgroups: $\mathrm{U}(3) \supset \mathrm{SU}(3) \supset$ $\mathrm{SO}(3)$, i.e. they are contracted with respect to the particle indices, then the system is invariant with respect to the transformations from one configuration to another. This set of transformation is the second pillar of MUSY.

A simple dynamically symmetric Hamiltonian characterized by this group-chain can describe the spectra of different configurations (obtained from different reactions) in a unified way [24], in spite of the fact that they are distributed in a large range of excitation energy and deformation. Occasionally even the prediction of the high-lying cluster spectra from the description of the well-established low-lying quartet (shell) spectrum is possible.

The spin-isospin degrees of freedom are handled in the particle scheme again in terms of Wigner's $\mathrm{U}^{\mathrm{ST}}(4) \supset \mathrm{U}^{\mathrm{S}}(2) \otimes \mathrm{U}^{\mathrm{T}}(2)$, symmetry, which is obtained in this case by the contraction of the $\mathrm{U}(4 \mathrm{n})$ total symmetry group. And the total antisymmetry requirement is formulated by the relation of the permutation groups of $S(n)$, which are contained in the subgroup-chain of the $U(n)$ particle symmetry groups both in the space and in the spin-isospin parts [22,23].

MUSY, as it is presented here, is a result of a multi step evolution. First it was invented for connecting different cluster configurations [25]. Therefore, it was also called multichannel dynamical symmetry, referring to the reaction channels that define the cluster configurations. The connection between the different configurations was found on the basis of wavefunction and energy eigenvalue arguments. The explicit transformations between the different configurations was established first for the case of two binary cluster configurations (in terms of Talmi-Moshinsky transformations) [26]. Then it was realized that the quartet configuration in particular, and the shell configuration in general [27], has a relation to the cluster configurations, which is similar to the one existing among them [28]. The present formulation of MUSY is a general one, incorporating any shell or cluster configuration. 
J. Cseh

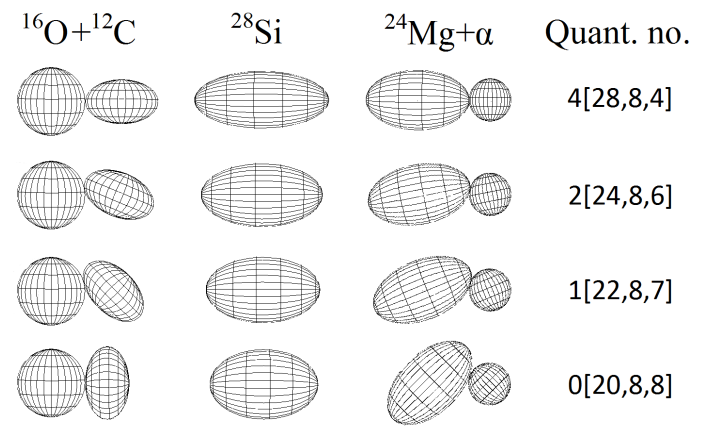

Figure 1. Cluster-shell duality in ${ }^{28} \mathrm{Si}$. The quantum numbers in parenthesis are the $\mathrm{U}(3)$ labels, while the first integer shows the major shell excitation quanta.

\subsection{Cluster-shell duality}

The cluster-shell duality is a historical and evergreen problem in structure studies. MUSY has some interesting contribution to this topic. The fact that shell and various cluster configurations can be identical with each other, as a consequence of the antisymmetrization, have been known for a long time. So far, however, it was discussed mainly in the low-energy region. MUSY-based studies, which apply large model space revealed several examples, when the quartet (shell) configuration has a 100\% overlap with several cluster configurations.

Especially remarkable are the cases of shape isomers. In particular, they correspond to extremely deformed shapes, where the $\mathrm{U}(3)$ symmetry is very stable [29]; sometimes even more than in the ground state. These special shell configurations usually have a single multiplicity, therefore, in the expansion of the cluster wavefunction only a single term appears, i.e. the two wavefunctions are identical. If it happens with several cluster configurations, they are also identical. In spite of the very different physical picture behind the shell and cluster model their states may have $100 \%$ overlap, as a consequence of the antisymmetrization.

Figure 1 shows an illustrative example. Strongly deformed states of the ${ }^{28} \mathrm{Si}$ nucleus are displayed with $0,1,2$, and 4 excitation quanta. The latter one is the newly predicted [30] and observed [31] superdeformed state.

\section{Summary and Conclusions}

In this contribution I introduced the multiconfigurational dynamical symmetry, which is the common intersection of the shell, collective and cluster models for the multi-major-shell problem. It is a composite symmetry in the sense that each configuration has a simple dynamical symmetry, and a further symmetry 
connects the configurations.

The previous one is a direct extension of the U(3) symmetry of the Elliott model: $\mathrm{U}_{s}(3) \otimes \mathrm{U}_{e}(3) \supset \mathrm{U}(3) \supset \mathrm{SU}(3) \supset \mathrm{SO}(3)$, found in the symplectic, contracted symplectic, and semimicroscopic algebraic cluster models. The representation labels of these groups provide us with a unified classification scheme of the shell, collective and cluster multiplets. The second symmetry, connecting the configurations, is the invariance with respect to the transformations in he pseudo space of the particle indices.

Due to the fact that MUSY connects the fundamental structure models, it is able to describe spectra of different configurations (from different reactions) distributed in a large range of excitation energy and deformation in a unified way. It may be ale to give occasionally interesting predictions, some of which have already been approved by experimental observation [24,32].

Not only the energy spectrum can be calculated from MUSY, but a new method for the determination of the shape isomers was also found, which is based on the stability and selfconsistency study of the connecting U(3) symmetry [32].

MUSY shows a dual symmetry breaking, similarly to the Elliott model: the U(3) and SU(3) symmetries are dynamically broken by some interactions expressed in terms of the invariant operators of their subgroups. On the other hand $\mathrm{SO}(3)$ is spontaneously broken in the eigenvalue problem of the intrinsic Hamiltonian, resulting in deformed shape. The deformed shapes look different from the shell and cluster perspectives, but sometimes the antisymmetrization may result in a complete overlap of their wavefunctions.

Some of the early applications of MUSY seem to suggest that this interesting symmetry is realized in some nuclei, nevertheless, it is still an open question, in which territory and to what extent is it a good approximation.

\section{Acknowledgements}

This work was supported by the National Research, Development and Innovation Fund of Hungary, financed under the K18 funding scheme with project no. K 128729.

\section{References}

[1] J.P. Elliott (1958) Proc. Roy. Soc. A 245 128; 562.

[2] K. Wildermuth, Th. Kanellopoulos (1958) Nucl. Phys. 7150.

[3] B.F. Bayman, A. Bohr (1958/59) Nucl. Phys. 9596.

[4] E. P. Wigner (1937) Phys. Rev. 51106.

[5] J.P. Draayer (1993) Fermion Models. In: R.F. Casten (ed) "Algebraic Approaches to Nuclear Structure" (Harwood Academic Publishers).

[6] D.J. Rowe (1985) Rep. Prog. Phys. 481419. 
[7] P.G. Reinhard, E.W. Otten (1984) Nucl. Phys. A 420173.

[8] W. Nazarewicz (1993) Int. J. Mod. Phys. E 2 Supp. 51.

[9] F. Iachello, A. Arima (1987) "The Interacting Boson Model" (Cambridge University Press, Cambridge).

[10] M.W. Kirson, A. Leviatan (1985) Phys. Rev. Lett. 552846.

[11] J. Cseh (2019) Phys. Lett. B 79359.

[12] K. Huang (2007) "Fundamental Forces of Nature" (World Scientific, Singapore).

[13] J.P. Elliott, P.G. Dawber (1986) "Symmetry in Physics" MacMillen Press, Chippenham).

[14] J. Cseh (2020) Eur. Phys. J. A Spec. Top. 2292543.

[15] G. Rosensteel, D.J. Rowe (1977) Phys. Rev. Lett. 38 10; (1980) Ann. Phys. (N.Y.) 126343.

[16] D.J. Rowe (1985) Rep. Prog. Phys. 481419.

[17] D.J. Rowe, G. Rosensteel (1982) Phys. Rev. C 25 3236(R).

[18] O. Castanos, J.P. Draayer (1989) Nucl. Phys. A 491349.

[19] F. Iachello (1981) Phys. Rev. C 23, 2778;

F. Iachello, R.D. Levine (1982) J. Chem. Phys. 773046.

[20] J. Cseh (1992) Phys. Lett. B 281173

[21] J. Cseh, G. Lévai (1994) Ann. Phys. (NY) 230165.

[22] J. Cseh (2021) Phys. Rev. C 103064322.

[23] P. Kramer, M. Moshinsky (1968) Group Theory of Harmonic Oscillators and Nuclear Structure. In: E.M. Loebl (ed) "Group Theory and Its Applications" (Academic Press, New York) p. 339.

[24] G. Riczu. J. Cseh (2021) Bulg. J. Phys. 48524.

[25] J. Cseh (1994) Phys. Rev. C 502240.

[26] J. Cseh, K. Kato (2013) Phys. Rev. C 87067301.

[27] J. Cseh (2015) Phys. Lett. B 743213.

[28] J. Cseh, G. Riczu (2016) Phys. Lett. B 757312.

[29] J. Cseh, G. Riczu, J. Darai (2019) Phys. Lett. B 79359.

[30] J. Darai, J. Cseh, D.G. Jenkins (2012) Phys. Rev. C 86064309.

[31] D.G. Jenkins et al (2012) Phys. Rev. C 86064308.

[32] J. Darai, contribution to this workshop. 